\title{
Covariant renormalizable gravity and its FRW cosmology
}

\author{
Shin'ichi Nojiri ${ }^{1}$ and Sergei D. Odintsov ${ }^{2 *}$ \\ ${ }^{1}$ Department of Physics, Nagoya University, Nagoya 464-8602, Japan \\ ${ }^{2}$ Instituciò Catalana de Recerca $i$ Estudis Avançats (ICREA) and Institut de Ciencies de l'Espai (IEEC-CSIC), \\ Campus UAB, Facultat de Ciencies, Torre C5-Par-2a pl, E-08193 Bellaterra (Barcelona), Spain
}

\begin{abstract}
We consider the diffeomorphism invariant gravity coupled with the ideal fluid in the non-standard way. The Lorentz-invariance of the graviton propagator in such a theory considered as perturbation over flat background turns out to be broken due to the non-standard coupling with the ideal fluid. As a result the behavior of the propagator in the ultraviolet/infrared region indicates that some versions of such theory are (super-)renormalizable ones (with appearance of only physical transverse modes). The FRW cosmology in same cases may be different from the one in General Relativity with the possible power law inflationary stage.
\end{abstract}

PACS numbers: $95.36 .+\mathrm{x}, 98.80 . \mathrm{Cq}$

Gravity theory belongs to special class among the four fundamental interactions since the corresponding quantum field theory has not been constructed. The main problem is that if we consider the perturbation from the flat background, which has a Lorentz invariance, by using General Relativity, there appear the non-renormalizable divergences coming from the ultraviolet region in momentum space. The superstring theories seem to be an almost unique candidate for the theory of quantum gravity. It is interesting how the ultraviolet behavior could be effectively modified in the superstrings theory. If the behavior of the graviton propagator in the ultraviolet region could be changed from $1 / k^{2}$ to $1 / k^{4}$, the theory could become ultraviolet renormalizable. Here $k^{2}$ is the square of the four momenta. Such a change, however, necessarily breaks the unitarity, since the action must include the higher derivatives with respect to the time coordinate as in case of higher derivative gravity (see book [1] for a general review).

The idea proposed in ref.[2] (for the extension and cosmological applications, see [3]) is to modify the ultraviolet behavior of the graviton propagator in Lorentz non-invariant way as $1 /|\boldsymbol{k}|^{2 z}$, where $\boldsymbol{k}$ is the spacial momenta and $z$ could be 2, 3 or larger integers. We define $z$ by the scaling properties of space and time coordinates $(\boldsymbol{x}, t)$ as follows,

$$
\boldsymbol{x} \rightarrow b \boldsymbol{x}, \quad t \rightarrow b^{z} t
$$

When $z=3$, the theory is UV renormalizable. Then in order to realize the Lorentz non-invariance, one introduces the terms breaking the Lorentz invariance explicitly (or more precisely, breaking full diffeomorphism invariance) by treating the temporal coordinate and the spacial coordinates in an anisometric way. Such model has the diffeomorphisms with respect only to the time coordinate $t$ and spacial coordinates $\boldsymbol{x}$ :

$$
\delta x^{i}=\zeta^{i}(t, \boldsymbol{x}), \quad \delta t=f(t) .
$$

Here $\zeta^{i}(t, \boldsymbol{x})$ and $f(t)$ are arbitrary functions. Recently, several works [4] criticized the model of ref.[2] (see also [3] ). They claimed that unphysical modes could appear in the theory. Such an appearance could occur due to the lack of the full diffeomorphism invariance. The local symmetry usually puts constraints on the system and only the physical modes appear as propagating modes. However, since above theory has not full diffeomorphism invariance, we might not be able to obtain full constraints to restrict the possible modes to the physical modes. Then in order to obtain consistent gravity theory, one usually needs the theory with full diffeomorphism invariance. Due to the lack of the full diffeomorphism invariance, we cannot, for example, choose the lapse function $N$ to be unity if $N$ depends on the spacial coordinates. In order to avoid the problem, Hořava [2] has assumed that the lapse function $N$ only depends on the time coordinate but not on the spacial coordinate. Due to the assumption, we do not obtain the local Hamilton constraint but only global one which requires that only the spacial integration of the Hamiltonian should vanish. It is not so clear if such a weak constraint can exclude all the unphysical modes or not. The problem does not seems to be settled.

In this note, we propose Hořava-like gravity model with full diffeomorphism invariance. When we consider the perturbations from the flat background, which has Lorentz invariance, the Lorentz invariance of the propagator is dynamically broken by the non-standard coupling with a perfect fluid. The obtained propagator behaves as $1 / \boldsymbol{k}^{2 z}$ with $z=2,3, \cdots$ in the ultraviolet region and the model could be perturbatively power counting (super-)renormalizable if $z>2$. Not as in the model [2], where unphysical modes might appear [4], only physical transverse modes appear

\footnotetext{
* Also at Center of Theor. Physics, TSPU, Tomsk
} 
in our model due to the full diffeomorphism invariance. The FRW dynamics of our model is also discussed. When $z \geq 3$, the FRW dynamics does not change from the Einstein gravity but when $z=2$, we obtain the modified FRW dynamics, where power law inflation as in quintessence or phantom like model, could occur.

Let us start from the Lorentz invariant action, or the action with full diffeomorphism invariance, where a perfect fluid couples with gravity in non-standard way, but due to the coupling, the effective Lorentz non-invariant action can be obtained. The fluid could not correspond to the usual fluid like, radiation, baryons, and dark matter, etc. As we discuss later, the non-standard fluid may come from the higher excited modes in string theories.

The starting action is

$$
S=\int d^{4} x \sqrt{-g}\left\{\frac{R}{2 \kappa^{2}}-\alpha\left(T^{\mu \nu} R_{\mu \nu}+\beta T R\right)^{2}\right\} .
$$

Here $T_{\mu \nu}$ is the energy-momentum tensor of the fluid. The action (3) is fully diffeomorphism invariant and belongs to general class of modified gravities (for review, see [5]). We consider the perturbation from the flat background $g_{\mu \nu}=\eta_{\mu \nu}+h_{\mu \nu}$. Then the curvatures have the following form:

$$
R_{\mu \nu}=\frac{1}{2}\left[\partial_{\mu} \partial^{\rho} h_{\nu \rho}+\partial_{\nu} \partial^{\rho} h_{\mu \rho}-\partial_{\rho} \partial^{\rho} h_{\mu \nu}-\partial_{\mu} \partial_{\nu}\left(\eta^{\rho \sigma} h_{\rho \sigma}\right)\right], \quad R=\partial^{\mu} \partial^{\nu} h_{\mu \nu}-\partial_{\rho} \partial^{\rho}\left(\eta^{\rho \sigma} h_{\rho \sigma}\right)
$$

The following gauge condition is chosen:

$$
h_{t t}=h_{t i}=h_{i t}=0 \text {. }
$$

Then the curvatures (5) have the following form:

$$
\begin{aligned}
& R_{t t}=-\frac{1}{2} \partial_{t}^{2}\left(\delta^{i j} h_{i j}\right), \quad R_{i j}=\frac{1}{2}\left\{\partial_{i} \partial^{k} h_{j k}+\partial_{j} \partial^{k} h_{i k}+\partial_{t}^{2} h_{i j}-\partial_{k} \partial^{k} h_{i j}\right\}, \\
& R=\partial^{i} \partial^{j} h_{i j}+\partial_{t}^{2}\left(\delta^{i j} h_{i j}\right)-\partial_{k} \partial^{k}\left(\delta^{i j} h_{i j}\right) .
\end{aligned}
$$

For the perfect fluid, the energy-momentum tensor in the flat background has the following form:

$$
T_{t t}=\rho, \quad T_{i j}=p \delta_{i j}=w \rho \delta_{i j} .
$$

Here $w$ is the equation of state $(\mathrm{EoS})$ parameter. Then one finds

$$
\begin{aligned}
& T^{\mu \nu} R_{\mu \nu}+\beta T R \\
& =\rho\left[\left\{-\frac{1}{2}+\frac{w}{2}+(-1+3 w) \beta\right\} \partial_{t}^{2}\left(\delta^{i j} h_{i j}\right)+(w-\beta+3 w \beta) \partial^{i} \partial^{j} h_{i j}+(-w+\beta-3 w \beta) \partial_{k} \partial^{k}\left(\delta^{i j} h_{i j}\right)\right]
\end{aligned}
$$

If we choose

$$
\beta=-\frac{w-1}{2(3 w-1)},
$$

the second term in the action (3) has the following form

$$
\alpha\left(T^{\mu \nu} R_{\mu \nu}+\beta T R\right)^{2}=\alpha \rho^{2}\left(\frac{w}{2}+\frac{1}{2}\right)^{2}\left\{\partial^{i} \partial^{j} h_{i j}-\partial_{k} \partial^{k}\left(\delta^{i j} h_{i j}\right)\right\}^{2}
$$

which does not contain the derivative with respect to $t$ and breaks the Lorentz invariance. We now assume $\rho$ is almost constant. Then in the ultraviolet region, where $\boldsymbol{k}$ is large, the second term in the action (3) gives the propagator behaving as $1 /|\boldsymbol{k}|^{4}$, which renders the ultraviolet behavior (compared with Eq.(1.4) in [2]).

Instead of (5), one may choose covariant gauge condition although we may need the ghost fields to keep the unitarity:

$$
\partial^{\mu} h_{\mu \nu}=0 \text { or } \partial^{t} h_{t \nu}=-\partial^{i} h_{i \nu}
$$

Then instead of (8), it follows

$$
\begin{aligned}
& T^{\mu \nu} R_{\mu \nu}+\beta T R \\
& =\rho\left[\left\{-\frac{1}{2}+\frac{w}{2}+(-1+3 w) \beta\right\}\left\{\partial_{t}^{2}\left(\delta^{i j} h_{i j}\right)+\partial_{k} \partial^{k} h_{t t}\right\}+(1+\beta-3 w \beta) \partial_{t}^{2} h_{t t}\right. \\
& \left.\quad+\left(-\frac{w \alpha}{2}-\beta+3 w \beta\right) \partial_{k} \partial^{k}\left(\delta^{i j} h_{i j}\right)\right]
\end{aligned}
$$


We should note the gauge condition (11) gives $\partial^{\mu} \partial^{\nu} h_{\mu \nu}=0$, that is,

$$
\partial_{t}^{2} h_{t t}=-\partial^{i} \partial^{j} h_{i j}-2 \partial^{i} \partial^{t} h_{t \partial_{i}}=\partial^{i} \partial^{j} h_{i j} .
$$

Then if imposing the condition identical with (9), we obtain (10), again.

Note that the form (9) indicates that the longitudinal mode does not propagate but only the transverse mode propagates. Not as in Hořava's model [2], where unphysical modes might appear [4], only physical transverse modes appear in our model due to the full diffeomorphism invariance in the action (3).

There are two special cases in the choice of $w$ : when $w=-1$, which corresponds to the cosmological constant, one gets $T^{\mu \nu} R_{\mu \nu}+\beta T R=0$ and therefore we do not obtain $1 / \boldsymbol{k}^{4}$ behavior. When $w=1 / 3$, which corresponds to the radiation or conformal matter, $\beta$ diverges and therefore there is no solution.

The apparent breakdown of the Lorentz symmetry in (10) occurs due to the coupling with the perfect fluid. The action (3) is invariant under the diffeomorphism in four dimensions and the energy-momentum tensor $T_{\mu \nu}$ of the non-standard fluid in the action should transform as a tensor under the diffeomorphism. The existence of the fluid, however, effectively breaks the Lorentz symmetry, which is the equivalence between the different inertial frames of reference. Note that the expression (77) is correct in the reference frame where the fluid does not flow, or the velocity of the fluid vanishes. In other reference frames, there appear non-vanishing $T_{i t}=T_{t i}$ components and there could appear the derivative with respect to time, in general. This situation is a little bit similar to the vacuum solution of the Einstein gravity, which is, of course, fully diffeomorphism invariant, but the obtained Schwarzschild solution is not Lorentz invariant. This is a kind of spontaneous breakdown of the symmetry, which is popular in the quantum field theory. The spontaneous breakdown of the symmetry is the phenomenon that the symmetry in the action is not realized in the vacuum. In the field theory, we consider the quantum theory starting from the vacuum with the broken symmetry. Similarly we may consider the quantum theory of the gravity from the background where the non-standard fluid exists and breaks the Lorentz symmetry. Then we may obtain (power-counting) renormalizable quantum gravity.

In the arguments after (4), we have assumed the flat background but the arguments could be generalized in the curved background: Even in the curved spacetime, due to the principle of the general relativity, we can always choose the local Lorentz frame. The local Lorentz frame has (local) Lorentz symmetry. Even in the local Lorentz frame, the perfect fluid might flow and $T_{i t}=T_{t i}$ components might not vanish. By boosting the frame, which is the (local) Lorentz transformation, we have a special local Lorentz frame, where the fluid does not flow. In the Lorentz frame, we can use the above arguments and find there is no breakdown of the unitarity. Conversely, in a general coordinate frame, $T^{\mu \nu} R_{\mu \nu}+\beta T R$ can have a derivative with respect to time.

We should also note that the action (3) admits the flat spacetime solution although there is a perfect fluid expressed by $T_{\mu \nu}$. Especially as a vacuum solution, there are Schwarzschild and Kerr black hole solutions.

The action (3) gives $z=2$ theory. In order that the theory could be ultra-violet power counting renormalizable in $3+1$ dimensions, we need $z=3$ theory. In order to obtain such a theory we note that, for any scalar quantity $\Phi$, the explicit forms of the covariant derivatives have the following form:

$$
T^{\mu \nu} \nabla_{\mu} \nabla_{\nu} \Phi+\gamma T \nabla^{\rho} \nabla_{\rho} \Phi=\rho\left[\{-1+(-1+3 w) \gamma\} g^{t t} \partial_{t}^{2} \Phi+\{w+(-1+3 w) \gamma\} \partial_{k} \partial^{k} \Phi\right]
$$

with a constant $\gamma$. Then if we choose

$$
\gamma=\frac{1}{3 w-1}
$$

one obtains

$$
T^{\mu \nu} \nabla_{\mu} \nabla_{\nu} \Phi+\gamma T \nabla^{\rho} \nabla_{\rho} \Phi=\rho(w+1) \partial_{k} \partial^{k} \Phi,
$$

which does not contain the derivative with respect to time coordinate $t$. This is true even if the coordinate frame is not local Lorentz frame. The derivative with respect to time coordinate $t$ is not contained in any coordinate frame, where the perfect fluid does not flow. Then if we consider

$$
S=\int d^{4} x \sqrt{-g}\left\{\frac{R}{2 \kappa^{2}}-\alpha\left(T^{\mu \nu} R_{\mu \nu}+\beta T R\right)\left(T^{\mu \nu} \nabla_{\mu} \nabla_{\nu}+\gamma T \nabla^{\rho} \nabla_{\rho}\right)\left(T^{\mu \nu} R_{\mu \nu}+\beta T R\right)\right\},
$$

with

$$
\beta=-\frac{w-1}{2(3 w-1)}, \quad \gamma=\frac{1}{3 w-1}
$$


we obtain $z=3$ theory, which is renormalizable. If one considers

$$
S=\int d^{4} x \sqrt{-g}\left[\frac{R}{2 \kappa^{2}}-\alpha\left\{\left(T^{\mu \nu} \nabla_{\mu} \nabla_{\nu}+\gamma T \nabla^{\rho} \nabla_{\rho}\right)\left(T^{\mu \nu} R_{\mu \nu}+\beta T R\right)\right\}^{2}\right],
$$

we obtain $z=4$ theory, which is super-renormalizable. In general, for the case

$$
S=\int d^{4} x \sqrt{-g}\left[\frac{R}{2 \kappa^{2}}-\alpha\left\{\left(T^{\mu \nu} \nabla_{\mu} \nabla_{\nu}+\gamma T \nabla^{\rho} \nabla_{\rho}\right)^{n}\left(T^{\mu \nu} R_{\mu \nu}+\beta T R\right)\right\}^{2}\right],
$$

with a constant $n$, we obtain $z=2 n+2$ theory. Usually $n$ should be an integer but in general, we may consider pseudo-local differential operator $\left(T^{\mu \nu} \nabla_{\mu} \nabla_{\nu}+\gamma T \nabla^{\rho} \nabla_{\rho}\right)^{n}$ with non-integer $n$ (e.g. $n=1 / 2,3 / 2$ etc.). We should also note that there are special cases, that is, $w=-1$ and $w=1 / 3$.

The second terms in the actions (3), (17), (19), and (20), which effectively break the Lorentz symmetry, are relevant only in the high energy/UV region since they contain higher derivative terms. In the IR region, these terms do not dominate and the usual Einstein gravity follows as a limit.

Forgetting the renormalizability and regarding the action (20) to be an effective action, we may consider negative $n$ case. In such a case, the action (20) represents the non-local theory.

We now briefly consider the cosmology. Starting from the FRW background with flat spacial part,

$$
d s^{2}=-d t^{2}+a(t)^{2} \sum_{i=1,2,3}\left(d x^{i}\right)^{2}
$$

it is easy to see that $T^{\mu \nu} \nabla_{\mu} \nabla_{\nu}+\gamma T \nabla^{\rho} \nabla_{\rho}$ only contains the derivative with respect to spacial coordinates. The extra terms in the actions (17), (19) and (20), which correspond to $z \geq 3$ case, vanishes if the metric of the spacial part is flat and we obtain the same FRW solutions as those in the Einstein gravity. The extra terms, however, could modify the perturbations from the FRW solution. There is, however, an exception corresponding to the action (3), that is, $z=2$ case. The $z=2$ model does not give power-counting renormalizable theory but it might express some effective theory. For example, we may consider the following type of very general action:

$$
S=\int d^{4} x \sqrt{-g}\left[\frac{R}{2 \kappa^{2}}-\sum_{n=0}^{N} \alpha_{n}\left\{\left(T^{\mu \nu} \nabla_{\mu} \nabla_{\nu}+\gamma T \nabla^{\rho} \nabla_{\rho}\right)^{n}\left(T^{\mu \nu} R_{\mu \nu}+\beta T R\right)\right\}^{2}\right] .
$$

Since the term with $n=N$ dominates in the very high energy region, we have $z=2 N+2$ theory, which is powercounting renormalizable. In low energy region, only the Einstein-Hilbert term dominates. However, in an intermediate energy region, $n=0$ term could dominate and effectively the action (3) follows. For the action (3), the extra term gives the modification from the Einstein gravity since the FRW universe is not the local Lorentz frame. In order to obtain the equation corresponding to the FRW equation, we assume the following form of the metric

$$
d s^{2}=-\mathrm{e}^{2 b(t)} d t^{2}+a(t)^{2} \sum_{i=1,2,3}\left(d x^{i}\right)^{2}
$$

Then the action (3) has the following form:

$$
S=\int d^{4} x a^{3}\left[\frac{\mathrm{e}^{-b}}{2 \kappa^{2}}\left(6 \dot{H}+12 H^{2}-6 \dot{b} H\right)-9 \alpha \rho^{2}(1+w)^{2} \mathrm{e}^{-3 b} H^{4}\right]
$$

The equation corresponding to the FRW equation can be obtained by putting $b=0$ after the variation over $b$ and it has the following form:

$$
\frac{3}{\kappa^{2}} H^{2}=\frac{27 \alpha \rho^{2}(1+w)^{2}}{2} H^{4}+\rho_{\text {matter }} .
$$

Here $\rho_{\text {matter }}$ is the usual matter energy density corresponding to radiation, baryons, and dark matter, etc. but not corresponding to the fluid appeared in the action (3). In the situation that the contribution from the matter could be neglected, there are two cases. One is trivial case $H=0$ and another is

$$
H^{2}=\frac{2}{9 \kappa^{2} \alpha(1+w)^{2} \rho^{2}} .
$$


This equation is consistent only if $\alpha$ is positive. Since the fluid corresponding to $\rho$ has a constant EoS parameter, we find $\rho=\rho_{0} a^{-3(1+w)}$ with a constant $\rho_{0}$. Then the solution of (26) is given by

$$
a(t) \propto\left(t_{0}-t\right)^{-1 / 3(1+w)},
$$

which is phantom like solution even if $w>-1$. Then there might occur the inflation due to this phantom like solution. The second term in (25) is relevant only if the curvature is rather large if we choose the parameter $\alpha$ properly. Then if the universe started with not so large curvature, the term becomes irrelevant and we obtain standard cosmology.

We now consider the case that $\alpha$ is negative. In this case, the matter energy density $\rho_{\text {matter }}$ in (25) can dominate if compared with the term coming from the Einstein part as $\rho_{\text {matter }} \gg \frac{3}{\kappa^{2}} H^{2}$ in the early universe. In such a case, $\rho_{\text {matter }}$ might express the energy density of the inflaton. If the matter corresponding to $\rho_{\text {matter }}$ has a constant EoS parameter $w_{\text {matter }}, \rho_{\text {matter }}$ behaves as $\rho_{\text {matter }}=\rho_{\text {matter } 0} a^{-3\left(1+w_{\text {matter }}\right)}$ with a constant $\rho_{\text {matter } 0}$. Then by neglecting the 1.h.s. of (25), we find

$$
\frac{27 \alpha \rho_{0}^{2}(1+w)^{2} a^{-6(1+w)}}{2} H^{4}+\rho_{\text {matter } 0} a^{-3\left(1+w_{\text {matter }}\right)} \sim 0
$$

which shows $H^{2} \propto a^{-3\left(1+w_{\text {matter }}\right) / 2+3(1+w)}=a^{3 / 2-3 w_{\text {matter }} / 2+3 w}$. Hence, the solution of (28) is given by

$$
a(t) \propto t^{2 / 3\left(1-w_{\text {matter }}+2 w\right)},
$$

if $1-w_{\text {matter }}+2 w>0$. On the other hand, if $1-w_{\text {matter }}+2 w<0$, we obtain

$$
a(t) \propto\left(t_{0}-t\right)^{2 / 3\left(1-w_{\text {matter }}+2 w\right)} .
$$

Eq.(29) describes quintessence-like inflation but Eq.(30) corresponds to the phantom-like one. Then depending on the values of $w_{\text {matter }}$ and $w$, both kinds of inflation could be realized.

Instead of the perfect fluid in (7), where the pressure $p$ is proportional to the energy density $\rho$, we can consider more general fluid, whose EoS is given by $p=f(\rho)$. Here $f(\rho)$ is general non-linear function of $\rho$. The cancellation of the time derivatives (arguments around (8)-(10)), which is necessary for the unitarity, however, only occurs when the EoS is linear equation. Then for general perfect fluid with $\operatorname{EoS} p=f(\rho)$, the cancellation does not occur or cannot be realized. The other types of the coupling should be considered in such a case.

In summary, we proposed gravity models with full diffeomorphism invariance, which has a renormalizable property as in Hořava's model of gravity. When we consider the perturbation from the flat background, which has Lorentz invariance, the Lorentz invariance of the propagator is dynamically broken by the non-standard coupling with the perfect fluid. As a result, the corresponding theory may be power counting (super-)renormalizable. Moreover, only physical transverse modes appear in our model due to the full diffeomorphism invariance. The FRW dynamics of such model is studied. When $z \geq 3$, the FRW cosmology does not change from the Einstein gravity cosmology. When $z=2$, we obtain the modified FRW dynamics, where quintessence/phantom-like inflation may occur.

We now speculate what could be the fluid which appears in the action (3). In order that the theory could be renormalizable, the fluid must be non-relativistic even in a very high energy regions, which is very unusual. In the string theory, however, there appear infinite number of heavy particles as excited states of string. As the energy becomes higher and higher, heavier and heavier particles are created by the quantum effects and the particles could make a non-relativistic fluid in any high energy region. Then this kind of fluid could naturally appear in the string theories.

As a final remark, let us note that it would be of interest to consider other dynamical scenarios of Lorentz invariance breaking in such a way that this invariance is restored at current dark energy epoch.

\section{Acknowledgments}

The work by S.N. is supported in part by Global COE Program of Nagoya University provided by the Japan Society for the Promotion of Science (G07). The work by S.D.O. is supported in part by MICINN (Spain) projects FIS2006-02842 and PIE2007-50I023 and by LRSS project N.2553.2008.2.

[1] I. L. Buchbinder, S. D. Odintsov and I. L. Shapiro, Effective Action In Quantum Gravity, IOP Publishing, 1992. 
[2] P. Horava, Phys. Rev. D 79, 084008 (2009) arXiv:0901.3775 [hep-th]].

[3] M. Visser, arXiv:0902.0590 [hep-th]; L. Maccione, A. M. Taylor, D. M. Mattingly and S. Liberati, JCAP 0904, 022 (2009) arXiv:0902.1756 [astro-ph.HE]]; P. Horava, arXiv:0902.3657] [hep-th]; A. Jenkins, arXiv:0904.0453 [gr-qc]; T. Takahashi and J. Soda, arXiv:0904.0554 [hep-th]; G. Calcagni, arXiv:0904.0829 [hep-th]; E. Kiritsis and G. Kofinas, arXiv:0904.1334 [hep-th]; J. Kluson, arXiv:0904.1343 [hep-th]; H. Lu, J. Mei and C. N. Pope, arXiv:0904.1595 [hep-th]; S. Mukohyama, arXiv:0904.2190 [hep-th]; R. Brandenberger, arXiv:0904.2835 [hep-th]; R. G. Cai, L. M. Cao and N. Ohta, arXiv:0904.3670 [hep-th]; H. Nikolic, arXiv:0904.3412 [hep-th]; H. Nastase, arXiv:0904.3604 [hep-th]; R. G. Cai, Y. Liu and Y. W. Sun, arXiv:0904.4104 [hep-th]; G. E. Volovik, arXiv:0904.4113 [gr-qc]; Y. S. Piao, arXiv:0904.4117 [hep-th]; X. Gao, arXiv:0904.4187 [hep-th]; E. O. Colgain and H. Yavartanoo, arXiv:0904.4357 [hep-th] S. Mukohyama, K. Nakayama, F. Takahashi and S. Yokoyama, arXiv:0905.0055 [hep-th]; R. G. Cai, B. Hu and H. B. Zhang, arXiv:0905.0255 [hep-th]; D. Orlando and S. Reffert, arXiv:0905.0301 [hep-th]; T. Ha, Y. Huang, Q. Ma, K. D. Pechan, T. J. Renner, Z. Wu and A. Wang, arXiv:0905.0396 [physics.pop-ph]; S. K. Rama, arXiv:0905.0700 [hep-th]; R. G. Cai, L. M. Cao and N. Ohta, arXiv:0905.0751 [hep-th]; R. H. Brandenberger, arXiv:0905.1514 [hep-th]; J. Kluson, arXiv:0905.1483 [hep-th]; R. A. Konoplya, arXiv:0905.1523 [hep-th]; J. W. Moffat, arXiv:0905.1668 [hep-th]; D. Blas, D. Comelli, F. Nesti and L. Pilo, arXiv:0905.1699 [hep-th]; S. Chen and J. Jing, arXiv:0905.2055 [gr-qc]; B. Chen, S. Pi and J. Z. Tang, arXiv:0905.2300 [hep-th]; J. Chen and Y. Wang, arXiv:0905.2786 [gr-qc]; T. P. Sotiriou, M. Visser and S. Weinfurtner, arXiv:0905.2798 [hep-th]; Y. W. Kim, H. W. Lee and Y. S. Myung, arXiv:0905.3423 [hep-th]; E. N. Saridakis, arXiv:0905.3532 [hep-th]; S. Mukohyama, arXiv:0905.3563 [hep-th]; G. Calcagni, arXiv:0905.3740 [hep-th]; A. Wang and Y. Wu, arXiv:0905.4117 [hep-th].

[4] C. Charmousis, G. Niz, A. Padilla and P. M. Saffin, arXiv:0905.2579 [hep-th]; M. Li and Y. Pang, arXiv:0905.2751 [hep-th].

[5] S. Nojiri and S. D. Odintsov, hep-th/0601213. 